\title{
Efficient butt-coupling of surface plasmons on a silver-air interface
}

\author{
Caitlin Fisher ${ }^{\mathrm{a}, *}$, Lindsay C. Botten ${ }^{\mathrm{b}, \mathrm{c}}$, Christopher G. Poulton ${ }^{\mathrm{c}}$, Ross C. \\ McPhedran $^{a}$, C. Martijn de Sterke ${ }^{a}$
${ }^{a}$ University of Sydney, Centre for Ultrahigh Bandwidth Devices for Optical Systems (CUDOS), School of Physics, Sydney, New South Wales, Australia, 2006
${ }^{\mathrm{b}}$ Australian National University, Centre for Ultrahigh Bandwidth Devices for Optical Systems (CUDOS), National Computational Infrastructure, Canberra, Australian Capital Territory, Australia, 0200
${ }^{c}$ University of Technology Sydney, Centre for Ultrahigh Bandwidth Devices for Optical Systems \\ (CUDOS), School of Physics, Sydney, New South Wales, Australia, 2006
}

\begin{abstract}
Butt-coupling of light into a surface plasmon is a simple and compact coupling method with a range of potential uses in photonic circuitry. Although butt-coupling has been successfully implemented in many coupling configurations, the coupling effectiveness is not fully understood. Here, we present a semi-analytical study which models the coupling efficiency of an incident beam into a surface plasmon on silver in the presence of loss using an projection method in one dimension. We find that the coupling efficiencies for silver between the wavelengths of $0.38-1.6 \mu \mathrm{m}$ reach $77-88 \%$ with optimum incident beam parameters.
\end{abstract}

Keywords: surface plasmons, butt-coupling, silver.

*Caitlin Fisher, caitlin.fisher@sydney.edu.au

\section{Introduction}

Surface plasmons (SPs) are collective field oscillations that propagate along the surface of a dielectric and a plasmonic material. The characteristics of SPs, such as propagation length and wavelength, are highly sensitive to the material properties of the interface ${ }^{1}$ and as such SPs are used to carry and store data in photonic circuitry, ${ }^{2}$ characterise surfaces, used in enhanced Raman spectroscopy ${ }^{3}$ and in highly sensitive biosensors ${ }^{45}$ and many more applications. Butt-coupling is a simple and compact method of exciting a SP by aiming a laser parallel to the interface. Unlike other excitation methods, butt-coupling does not require extra structures, such as prism, gratings or nanostructures, and instead of matching the parallel k-vectors of the incident beam and the SP, the coupling efficiency is determined to a large degree by the overlap between the incident and transmitted field profiles. 
While butt-coupling has been shown in theory to potentially reach maximum coupling efficiencies of $80-90 \%^{6}{ }^{7}$ in reality, these coupling efficiencies are difficult to achieve. Here, we aim to deepen our understanding of the fundamental butt-coupling mechanism between an incident beam and the SP and to determine the maximum coupling efficiencies possible. Here we model the butt-coupling mechanism for silver (Ag) using a projection method using the complex permittivities from the literature. ${ }^{8}$ This allows us to calculate the maximum SP coupling efficiencies possible by optimising the geometry of the incident beam.

In Sec. 2, we explain the model we use to determine the coupling efficiency. In Sec. 3, we present the coupling efficiencies for Ag for various incident beam geometries, and in Sec. 4 we close with some concluding remarks.

\section{Method}

The butt-coupling configuration is shown in Fig. 1: an incident Gaussian beam from $z<0$ (Region I) is aimed at the air-Ag interface in $z>0$ (Region II) and a SP propagating along the interface in the positive $z$-direction. The $\varepsilon_{d}$ and $\varepsilon_{m}$ are the relative permittivities of the air and $\mathrm{Ag}$ respectively. For the SP to exist, the field components $H_{y}, E_{x}$ and $E_{z}$ are transverse magnetic. The whole configuration is bounded by perfectly conducting waveguide walls at $x= \pm l$ for the analytical convenience of using discrete waveguide modes in our calculations.

To calculate the SP coupling efficiency, we first write the $H_{y}$ and $E_{x}$ fields in Region I and Region II as superpositions of waveguides modes propagating in the positive or negative $z$-direction. The $H_{y}$ fields in Region I and II are

$$
\begin{aligned}
H_{y}^{\mathrm{I}} & =\sum_{p}\left(\chi_{p}^{\mathrm{I}}\right)^{-\frac{1}{2}}\left[\alpha_{p} \exp \left(i \beta_{p}^{\mathrm{I}} z\right)+r_{p} \exp \left(-i \beta_{p} z\right)\right] \mathcal{H}_{p}^{\mathrm{I}}(x), \\
H_{y}^{\mathrm{II}} & =\sum_{q}\left(\chi_{q}^{\mathrm{II}}\right)^{-\frac{1}{2}} t_{q} \exp \left(i \beta_{q}^{\mathrm{II}} z\right) \mathcal{H}_{q}^{\mathrm{II}}(x)
\end{aligned}
$$

respectively, where $\beta$ are propagation constants, $\chi=\beta / k_{0}$ where $k_{0}$ is the wavevector in free space, and the fields have a $\exp (-i \omega t)$ time-dependence where $\omega$ is the angular frequency. The $\alpha, r$ and $t$ are the incident, reflection and transmission coefficients respectively. The $\mathcal{H}^{\mathrm{I}}(x)$ and $\mathcal{H}^{\mathrm{II}}(x)$ are waveguide modes, which satisfy the condition that the transverse electric field is zero at the waveguide walls, $E_{x}(x= \pm l)=0$, and for Region II, that the modes are continuous over the air-Ag interface $x=\Delta l$. For this condition to 


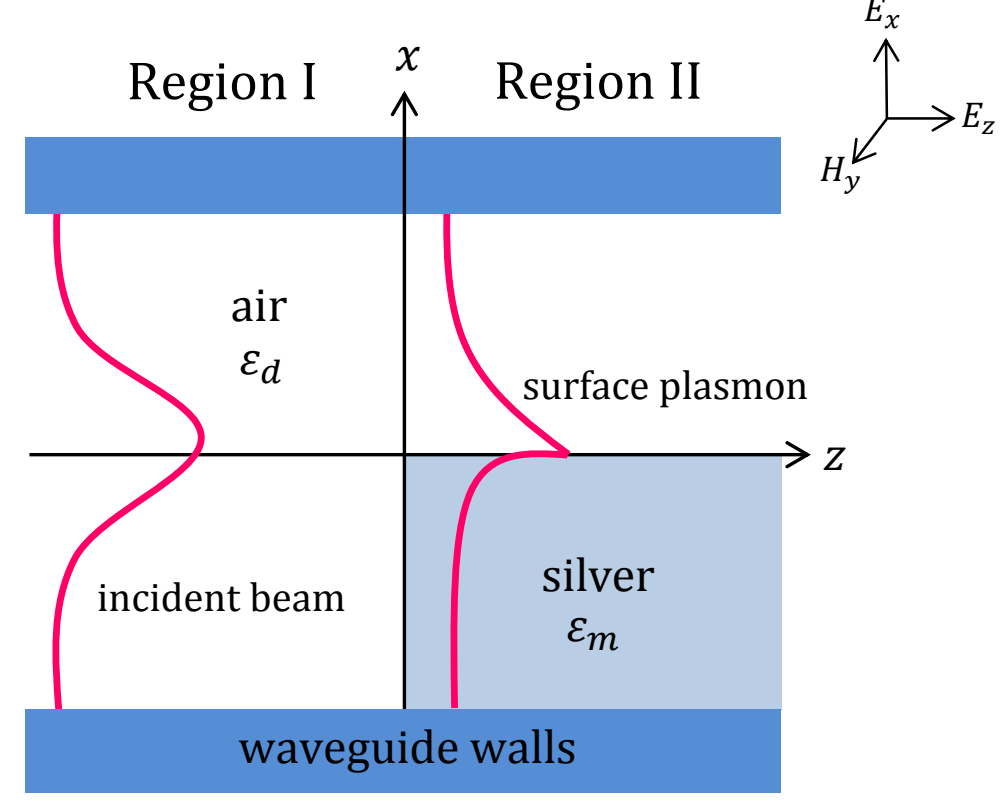

Fig 1: Butt-coupling configuration modelled in this work.

be satisfied, the $\beta_{q}^{\mathrm{II}}$ must satisfy the transcendental equation

$$
\frac{\gamma_{\mathrm{d}}}{\varepsilon_{\mathrm{d}}} \cosh \left(\gamma_{\mathrm{d}}(l-\Delta l)\right)+\frac{\gamma_{\mathrm{m}}}{\varepsilon_{\mathrm{m}}} \cosh \left(\gamma_{\mathrm{m}}(l+\Delta l)\right)=0
$$

where $\gamma_{\mathrm{d}}$ and $\gamma_{\mathrm{m}}$ are the air and silver transverse wavenumbers respectively. In this work, the $\beta^{\mathrm{II}}$ are found numerically.

By using Maxwell's equations, the $E_{x}$ fields for Region I and II can be derived from Eq. (1) and are

$$
\begin{aligned}
& E_{x}^{\mathrm{I}}=\sum_{p}\left(\chi_{p}^{\mathrm{I}}\right)^{-\frac{1}{2}}\left[\alpha_{p} \exp \left(i \beta_{p}^{\mathrm{I}} z\right)-r_{p} \exp \left(-i \beta_{p}^{\mathrm{I}} z\right)\right] \mathcal{E}_{p}^{\mathrm{I}}(x) \\
& E_{x}^{\mathrm{II}}=\sum_{q}\left(\chi_{q}^{\mathrm{II}}\right)^{-\frac{1}{2}} t_{q} \exp \left(i \beta_{q}^{\mathrm{II}} z\right) \mathcal{E}_{q}^{\mathrm{II}}(x) .
\end{aligned}
$$

where $\mathcal{E}(x)=Z_{0} \mathcal{H}(x) / \varepsilon$ for the appropriate region and $Z_{0}$ is the impedance of free space. $\mathcal{H}(x)$ and $\mathcal{E}(x)$ are normalised to satisfy the orthogonality condition

$$
\int_{-l}^{l} \mathcal{H}_{p}^{\mathrm{I}} \mathcal{E}_{p^{\prime}}^{\mathrm{I}} d x=\delta_{p p^{\prime}}
$$

and similarly for Region II, where $\delta$ is the Dirac delta function. 
The continuity conditions over the interface are $H_{y}^{\mathrm{I}}\left(z=0^{-}\right)=H_{y}^{\mathrm{II}}\left(z=0^{+}\right)$and $E_{x}^{\mathrm{I}}(z=$ $\left.0^{-}\right)=E_{x}^{\mathrm{II}}$. The incident fields are known i.e. the $\alpha$ coefficients are chosen initially, and the other fields have unknown $r$ and $t$ coefficients. To solve for $r$ and $t$, we use a projection method where the key idea is to expand each Region I mode by the set of Region II modes, and vice versa and using these projections to solve the continuity conditions. Only the fundamental results of the projection method are given here as detailed derivations are similar to those published earlier. ${ }^{9}$

The projections of each Region II mode onto the set of Region I modes, and vice versa, are stored in the $\boldsymbol{J}$ and $\boldsymbol{K}$ matrices where the $p q^{\text {th }}$ element of each are

$$
\begin{aligned}
J_{p q} & =\int_{-l}^{l} \mathcal{E}^{\mathrm{I}}(x) \mathcal{H}^{\mathrm{II}}(x) d x, \\
K_{p q} & =\int_{-l}^{l} \mathcal{H}^{\mathrm{II}}(x) \mathcal{E}^{\mathrm{I}}(x) d x
\end{aligned}
$$

respectively. It can be shown at full rank that $\boldsymbol{J}^{T} \boldsymbol{K}=\boldsymbol{I}$ and $\boldsymbol{J} \boldsymbol{K}^{T}=\boldsymbol{I}$, where $\boldsymbol{I}$ is an identity matrix of the appropriate size. These completeness relations show that each mode is perfectly represented by the infinite set of alternate modes.

Having implemented the projection method to solve the continuity conditions, the unknown coefficients solutions are

$$
\begin{array}{r}
\boldsymbol{r}=\left(\boldsymbol{I}+\boldsymbol{A} \boldsymbol{A}^{T}\right)^{-1}\left(\boldsymbol{A} \boldsymbol{A}^{T}-\boldsymbol{I}\right) \boldsymbol{\alpha}, \\
\boldsymbol{t}=2 \boldsymbol{A}^{T}\left(\boldsymbol{I}+\boldsymbol{A} \boldsymbol{A}^{T}\right)^{-1} \boldsymbol{\alpha},
\end{array}
$$

where $\boldsymbol{\alpha}, \boldsymbol{r}$ and $\boldsymbol{t}$ are column vectors of coefficients, and $\boldsymbol{A}=\left(\chi^{\mathrm{I}}\right)^{\frac{1}{2}} \boldsymbol{J}\left(\chi^{\mathrm{II}}\right)^{-\frac{1}{2}}$, where $\chi$ is a square matrix with $\chi$ values as the diagonal entries.

The energy flux for each region is defined as the Poynting vector integrated from $x= \pm l$, and the energy flux conservation condition at $z=0$ is $\boldsymbol{t}^{H}\left(\boldsymbol{X}^{\mathrm{II}}+\left(\boldsymbol{X}^{\mathrm{II}}\right)^{H}\right) \boldsymbol{t}$ where $\boldsymbol{X}$ is defined for convenience as $\boldsymbol{X}=(\boldsymbol{\chi})^{-\frac{1}{2}{ }^{H}} \boldsymbol{W}(\boldsymbol{\chi})^{\frac{1}{2}}$ for the appropriate region, where the elements of $\boldsymbol{W}$ are

$$
W_{p p^{\prime}}^{\zeta}=\int_{-l}^{l} \mathcal{H}_{p}^{\mathrm{I} *} \mathcal{E}_{p^{\prime}}^{\mathrm{I}} d x
$$

and simiarily for Region II. 
The SP coupling efficiency is defined as

$$
\eta=\frac{\left|t_{1}\right|^{2}}{\left(\sum_{p \in \Omega_{\mathrm{p}}^{\mathrm{I}}}\left|\alpha_{p}\right|^{2}-2 \operatorname{Im} \sum_{p \in \Omega_{\mathrm{e}}^{\mathrm{I}}} \alpha_{p} r_{p}^{*}\right)} .
$$

where the denominator is the total incident energy flux. ${ }^{9} \Omega_{\mathrm{p}}$ and $\Omega_{\mathrm{e}}$ are the set of propagating and evanescent Region I modes respectively.

\section{Results and Discussion}

Here, we present the SP coupling efficiencies for Ag in the wavelength range $0.38-1.6 \mu \mathrm{m}$ for the configuration shown in Fig. 1. The wavelength range was chosen to start from where the SP exists i.e. $\operatorname{Re}\left\{\varepsilon_{\mathrm{m}}\right\}<-\varepsilon_{\mathrm{d}}=-1$, and to span into the infrared region where the number of modes required for our calculations pushes our computational limits. The relative permittivity of $\mathrm{Ag}$ in this wavelength range $^{8}$ is shown in Fig. 2. The incident field $\mathrm{E}_{x}^{\mathrm{I}}$ is chosen to be a Gaussian at $z=0^{-}$with a peak amplitude of 1 at $x=l_{G}$ and full width $w$ at $1 / e$ of the peak, as depicted in Fig. 1. The FWHM of the intensity is $w \sqrt{\ln (2) / 2}$.
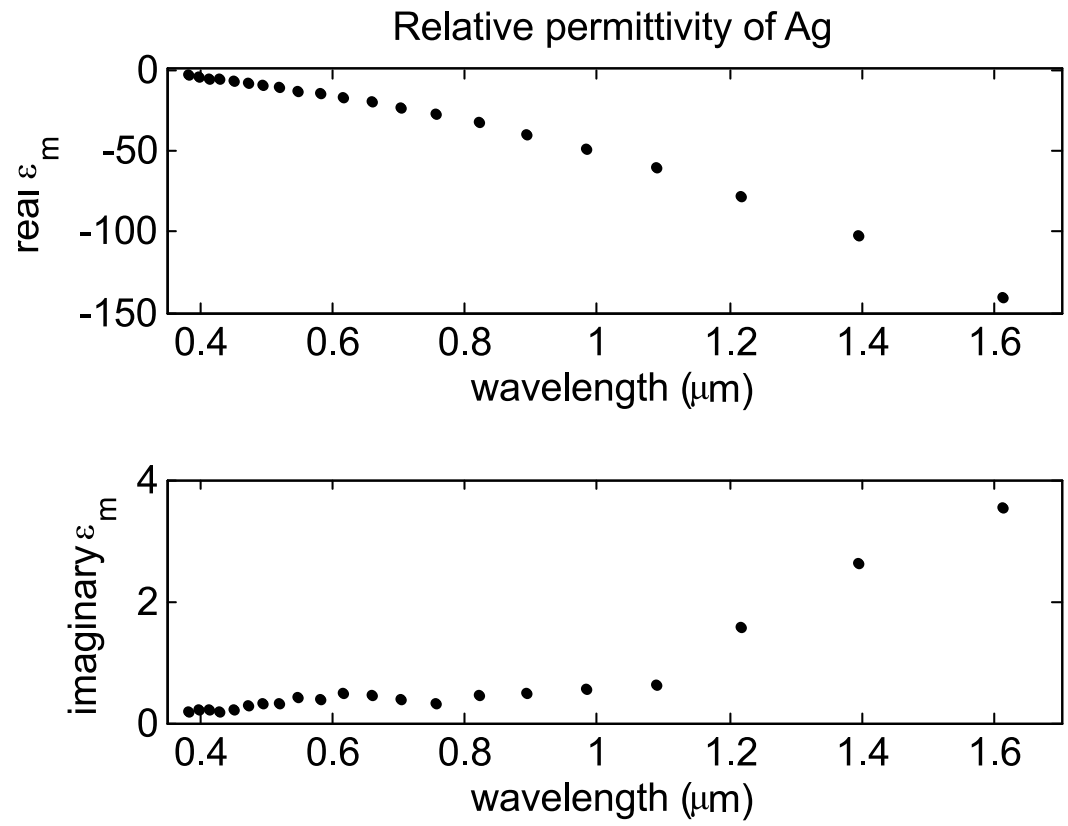

Fig 2: Relative permittivity of Ag from Johnson and Christy. ${ }^{8}$ 
We calculate the SP coupling efficiencies for various incident beam positions $l_{\mathrm{G}}$ and widths $w$. The maximum SP coupling efficiencies are given in Fig. 3(a) and the corresponding optimium $l_{\mathrm{G}}$ and $w$ are given in Fig. 3(b) and (c). The maximum coupling efficiencies reach $77-88 \%$ over the wavelength range. We have verified that the optimum $l_{\mathrm{G}}$ and $w$ are the values which maximise the field overlap between the incident beam and the SP mode. The step-like behaviour of the optimum $l_{\mathrm{G}}$ in Fig. 3(b) is due to the minimum grid spacing of our parameter sweep.

Figure 4 shows the total $\left|E_{x}\right|$ for Ag at wavelength $0.381 \mu \mathrm{m}$ with optimum incident beam parameters $l_{\mathrm{G}}=0.078 \mu \mathrm{m}$ and $w=0.191 \mu \mathrm{m}$. The white solid lines represent the corner of the Ag. Figure 4(a) is a close-up of the Ag corner where the incident beam comes in from the left $z<0$ and we see there is a point of high field density at the corner of the metal. The SP is visible propagating along the horizontal surface of the $\operatorname{Ag} z>0$, indicated by the presence of $E_{x}$ field penetrating the metal. The walls in this configuration are chosen to maximise the vertical surface of the Ag to minimise reflections off the waveguide walls. In this figure, the walls are at $l= \pm 10 \mu \mathrm{m}$ and $-l=-295 \mu \mathrm{m}$ which is more than $750 \lambda$, limited by the large number of waveguide modes and the corresponding computing power required. Figure 4(b) clearly shows no Fabry-Perot resonance along the vertical face of the Ag, suggesting that the artificial waveguide walls have little effect on the calculations. 

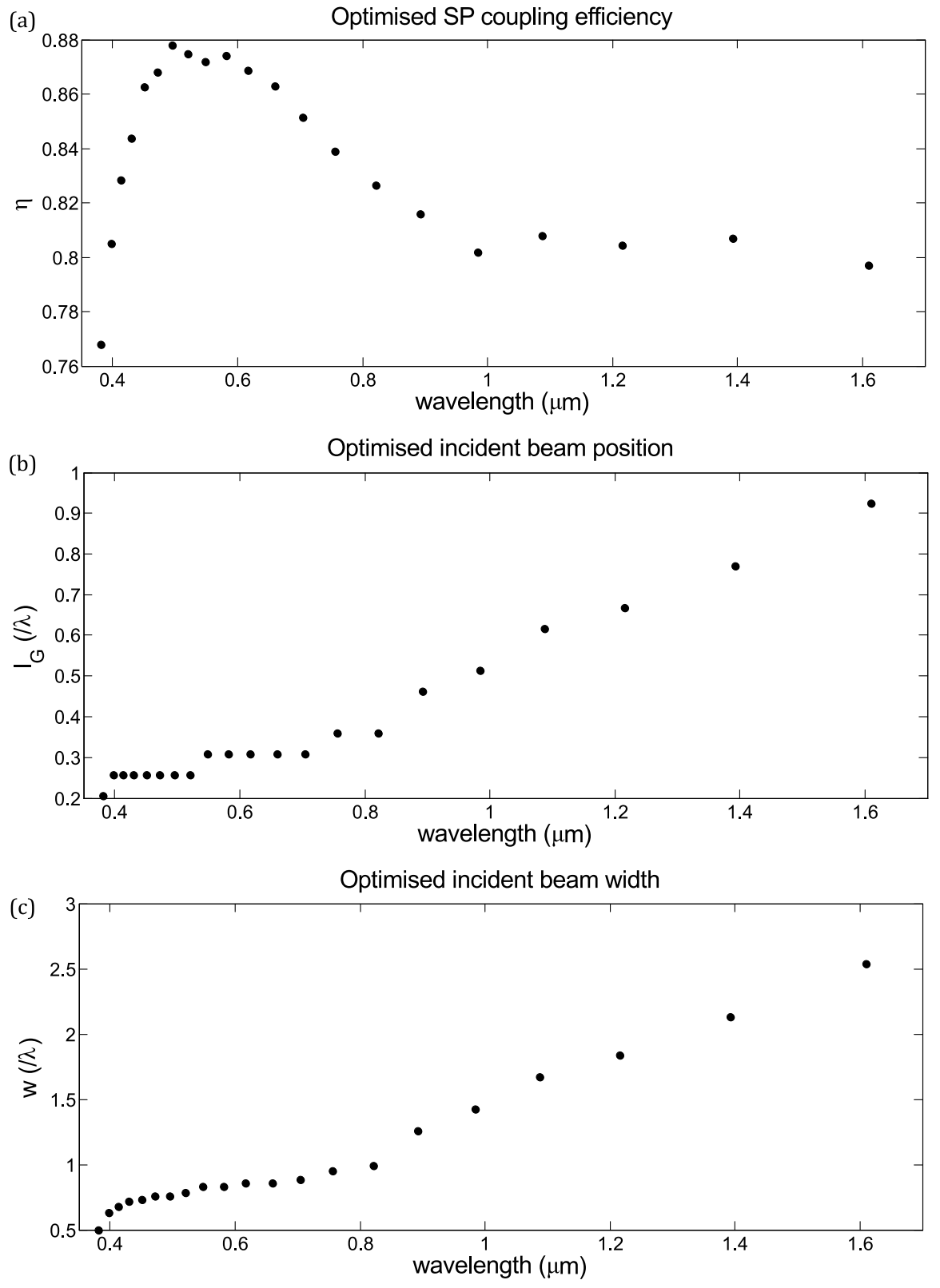

Fig 3: (a) Maximum SP coupling efficiencies for optimised incident beam parameters. The corresponding optimum incident parameters are also given: (b) optimum beam position $l_{\mathrm{G}}$, and (c) optimum beam width $w$. 

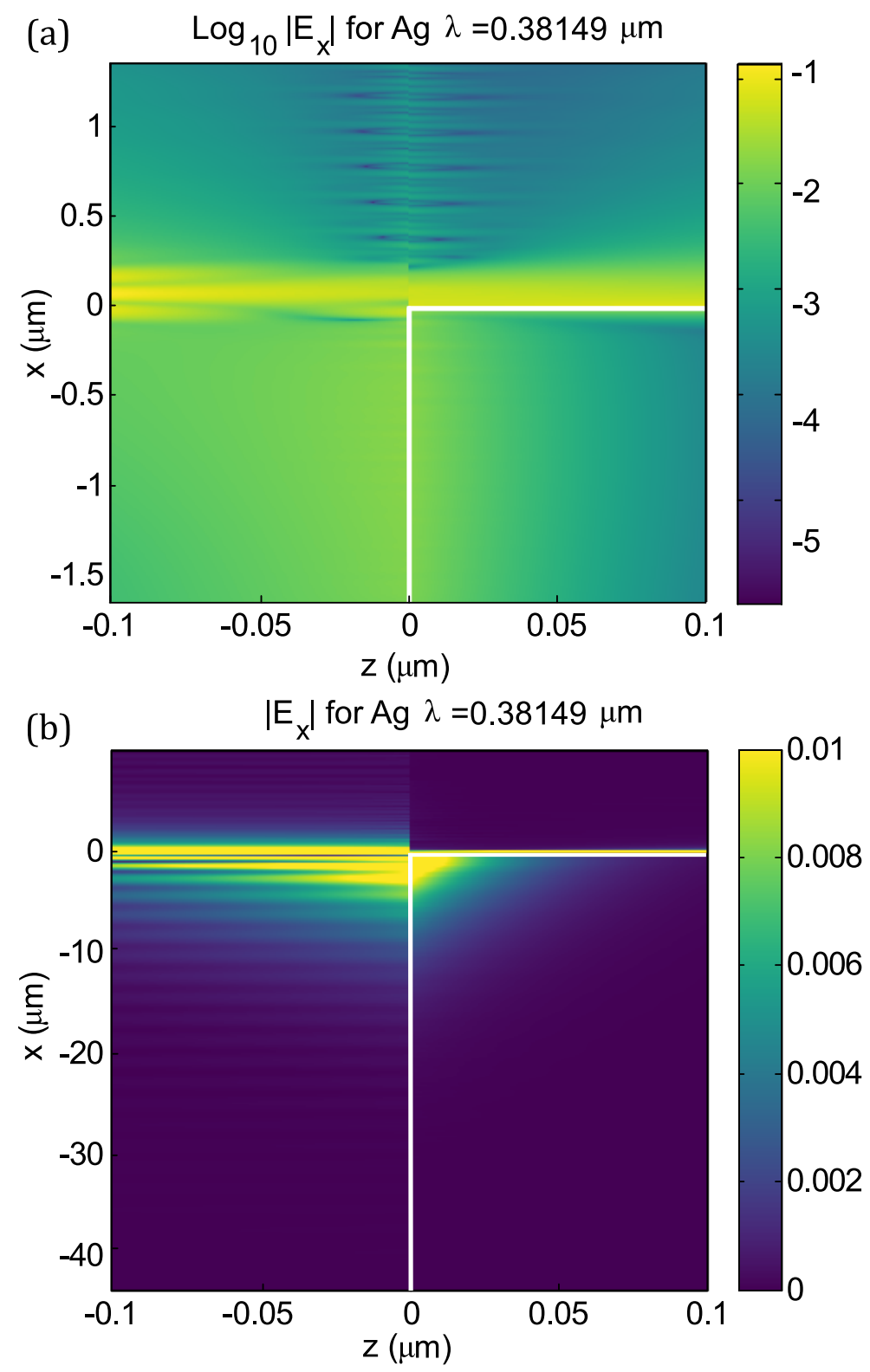

Fig 4: (a) $\log _{10}$ of the total $\left|E_{x}\right|$ field for $\mathrm{Ag}$ at $\lambda=0.3815 \mu \mathrm{m}$ at the corner, giving a close-up of the SP. (b) Total $\left|E_{x}\right|$ field over a larger area. The white lines for (a) and (b) mark the outline of the Ag. 


\section{Conclusion}

We have calculated the optimum SP coupling efficiencies for butt-coupling to Ag for a range of wavelengths $0.38-1.6 \mu \mathrm{m}$ by optimising the beam width and position of the incident Gaussian beam. The resulting coupling efficiencies range from $77-88 \%$ with best performance in the visible region around $0.4-0.8 \mu \mathrm{m}$. These high coupling efficiencies show the butt-coupling mechanism to be a good candidate for exciting SPs in photonic circuitry and many other applications.

\section{Acknowledgments}

This research was supported by the Australian Research Council (ARC) Centre of Excellence for Ultrahigh Bandwidth Devices for Optical Systems (CE110001018).

\section{References}

1 P. Berini, "Bulk and surface sensitivities of surface plasmon waveguides," New Journal of Physics 10, 105010 (2008).

2 J. Zhang, L. Zhang, and W. Xu, "Surface plasmon polaritons: physics and applications," Journal of Physics D: Applied Physics 45, 113001 (2012).

3 B. Sharma, R. R. Frontiera, A.-I. Henry, E. Ringe, and R. P. V. Duyne, "SERS: Materials, applications and the future," Materials Today 15, 16-25 (2012).

4 J. Homola, S. S. Yee, and G. Gauglitz, "Surface plasmon resonance sensors: review," Sensors and Actuators B - Chemical 54, 3-15 (1999).

5 S. Roh, T. Chung, and B. Lee, "Overview of the characteristics of micr- and nanostructured surface plasmon resonance sensors," Sensors 11, 1565-1588 (2011).

6 G. I. Stegeman, R. F. Wallis, and A. A. Maradudin, "Excitation of surface polaritons by end-fire coupling," Optics Letters 8, 386-8 (1983).

7 Z. Sun and D. Zeng, "Coupling of surface plasmon waves in metal/dielectric gap waveguides and single interface waveguides," Journal of the Optical Society of America B 24, 2883-7 (2007).

8 P. B. Johnson and R. W. Christy, "Optical constants of noble metals," Physical Review B 6, 4370-9 (1972).

9 C. Fisher, L. C. Botten, C. G. Poulton, R. C. McPhedran, and C. M. de Sterke, "Efficient end-fire coupling of surface plasmons in a metal waveguide," Journal of Optical Society of America B 32, 412-25 (2015). 